\title{
Research on User Integrated Energy Efficiency Evaluation Based on Balanced Score Card Method
}

\author{
Xudong Wang ${ }^{a}$, Jian Zhuang ${ }^{b}$, Leijiao Ge $e^{c^{*}}$, Hui Wang ${ }^{d}$, and Yanhe Liang ${ }^{e}$ \\ ${ }^{a}$ Dept. of Electric Power Research Institute, State Grid Tianjin Power Company, Tianjin, China \\ ${ }^{\mathrm{b}}$ School of Electrical Engineering and Automation, Tianjin University, Tianjin, 300072, China \\ ${ }^{c}$ Dept. of Development and Planning, State Grid Tianjin Power Company, Tianjin, 300010, China \\ ${ }^{\mathrm{d}}$ Beijing Electric Power Economic Research Institute, Beijing, 100055, China \\ ${ }^{\mathrm{e}}$ Dept. of Measurement Center, State Grid Heilongjiang Power Company, Heilongjiang, China \\ *Corresponding author: Leijiao Ge, legendglj99@tju.edu.cn
}

\begin{abstract}
Energy efficiency plays an important role in smart industrial park. The joint efforts of end users are useful to enhance the overall energy efficiency of smart industrial park. An improved balanced score card method for the assessment is proposed to guide the improvement of the integrated energy efficiency of end users. Firstly, the user integrated energy efficiency evaluation index is proposed from the fourth aspects of reliability, security, environmental benefits and flexibility. Secondly, it designs the balance score card (BSC) for the user integrated energy efficiency evaluation. In order to accelerate the calculation efficiency in BSC, an improved BSC method of the weight is proposed. Finally, a case verifies the feasibility of this method, and provides reference for the integrated energy efficiency evaluation of end users.
\end{abstract}

Keywords: formatting, style, styling, insert

\section{Introduction}

In smart industrial park, power users have a more power energy properties due to smart distribution system, which includes distribution generation (DGs), micro-grid (MGs), smart home, and flexible load and so on. At the same time, the energy property adds the characteristics of the mutual coupling, real-time interactive, complementary advantages among cooling, hot and electricity, because some advanced technologies are applied in smart industrial park, which include combined cooling heating and power (CCHP), geo-exchange systems, central air-conditioning system, ice storage central airconditioning system, storage system, etc. Then, the end users have many different choices in the comprehensive utilization of energy. From a short period of time, such as 1 minute, 1 second and so on, power energy of end user can choose the power grid, CCHP, or energy storage, etc. In a long period of time, such as 1 hour, 24 hours, etc., the end users will use a variety of energy in a variety of different time scale, in order to achieve the most energy efficiency by electric energy storage, heat storage, etc. Therefore, from the perspective of an energy supply enterprise, it is useful to assess the integrated energy efficiency of end users, in order to ensure the reasonable use of energy in the region, to provide data support in the region for energy dispatch and scientific energy, and to provide the basis for customers with customized energy services. And it needs to worth the further study.

There are many researches about to the integrated energy efficiency in international and domestic. In [1], it puts forward a multi adaptive planning system for regional power grid from four aspects of the performance, efficiency, social benefit and cost. The energy efficiency index of distribution system was constructed in [2]. Many Scholars have researched on energy efficiency, including the software platform [3], the construction of the index system [2,4,5,6,8], industry standard [9], subjective and objective assessment method [10,12,14], and the hybrid evaluation method [15], etc.. Generally, 
these studies are the pre-assessment of the project based on energy or grid planning, which are less involved in the assessment of energy supply processes.

The rest of this paper is organized as follows. The next section gives the integrated energy efficiency index system. In section 3, we present the balanced score card method for the weight of index system. Section 4 presents case studies to verify the advantages of the proposed BSC method. Finally, the paper concludes with the summary of the main points.

\section{Integrated Energy Efficiency Evaluation Index}

According to the international and domestic energy standards in smart industrial park, considering the usage habit of end user's energy and the principle of the easy calculation, the evaluation index is proposed from the fourth aspects of reliability, safety, environmental benefits and flexibility. It is shown in Fig. 1.

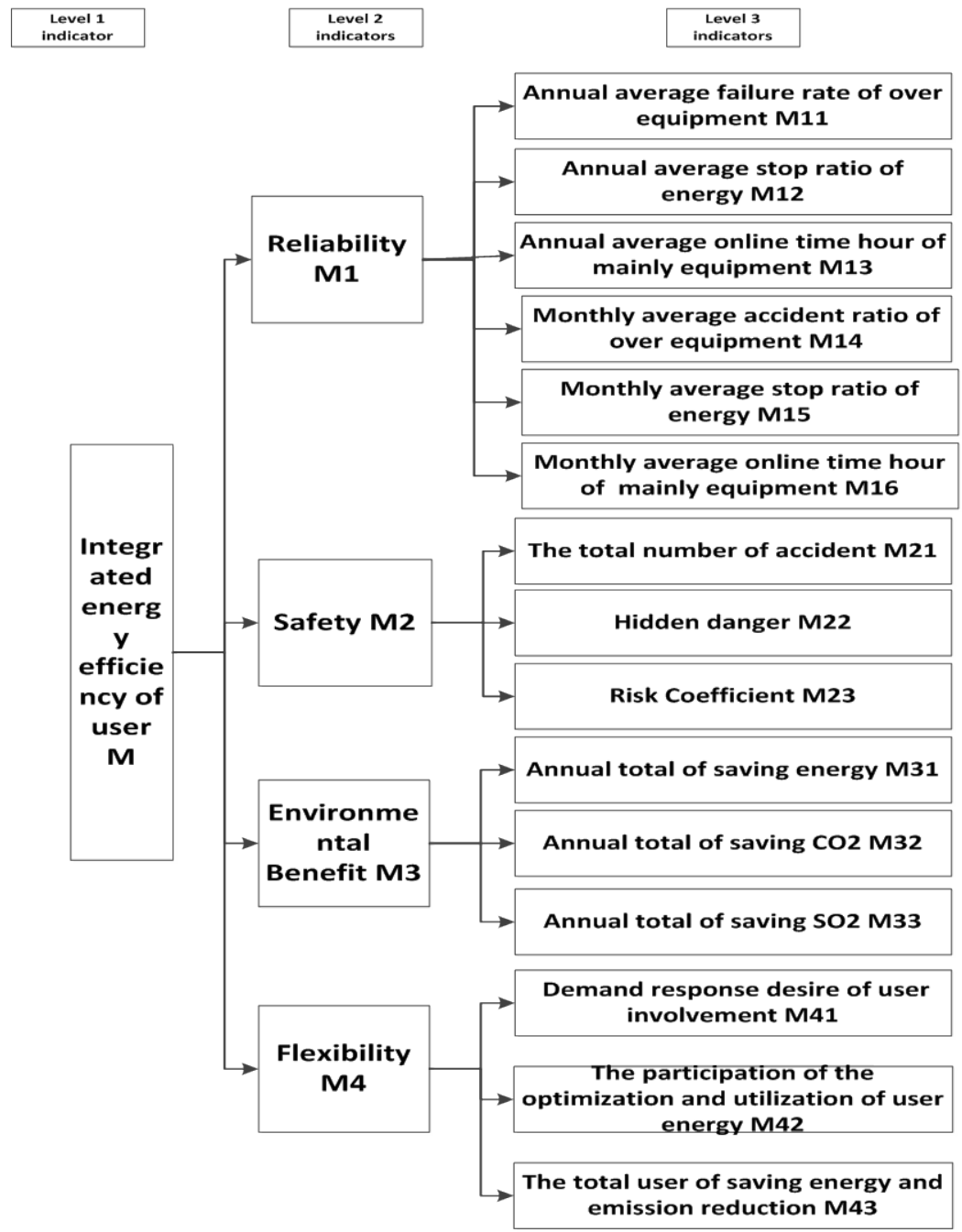

Fig. 1. Integrated energy efficiency evaluation index of end user

In order to calculate the index, considering the limit of paper page and some indicators have been well known, some key indicators of the index system are formulated as follow.

A Reliability M1

$$
M_{11}=\frac{B}{C} \times 100 \%
$$


Where M11 is an annual average failure rate of overall equipment. B is an annual failure time hours of overall equipment. $\mathrm{C}$ is some annual online time hours of over equipment.

$$
M_{12}=\frac{D}{E} \times 100 \%
$$

Where M12 is an annual average supply ratio of user energy. D is an annual stop time hours of user energy. $\mathrm{E}$ is an annual online time hours of user energy.

B Safety M2

$$
M_{23}=\left\{\begin{array}{c}
\text { High, } \quad \eta \geq 90 \% \\
\text { Normal, } 90 \%>\eta \geq 60 \%, \eta=\frac{F}{H} \times 100 \% \\
\text { Low, } \quad \eta<60 \%
\end{array}\right.
$$

Where M23 is a risk index. $\eta$ is a monthly accident rate of user. $\mathrm{F}$ is a total accident rate of user per month. $\mathrm{H}$ is a total number of user.

C Environmental benefits M3

$$
M_{31}=\sum_{i \in R} a_{i}
$$

Where M31 is an annual amount of saving energy. The ai is a saving energy of the i-th user in the region.

D Flexibility M4

$$
M_{42}=\frac{J}{K} \times 100 \% \quad(5)
$$

Where M42 is a participation degree of user energy optimization and utilization. $\mathrm{J}$ is a total number of participation of the user energy optimization and utilization in the region. $\mathrm{K}$ is an amount of the user in the region.

\section{Balanced Score Card}

Balanced Score Card (BSC) proposed by Robert Kaplan and David Norton Balanced in 1990s is a performance evaluation system. Its main idea is achieving the strategic planning of the enterprises through figures, tables, and cards. It includes four layer of financial, customer, internal business process, learning and growth. The financial layer is mainly reflected to the strategy and implementation of the enterprise, and to improve the enterprise's profit contribution. Customer layer is mainly reflected to the actual market size. The internal business process is the key internal management processes of the organization. The learning and growth is mainly reflected to the organization's long-term growth, and to improve corporate culture.

The integrated energy efficiency evaluation index of end user in smart industrial park includes four aspects of reliability, safety, environmental benefit and flexibility. The BSC consists of the financial, customer, internal business process, learning and growth. It builds a BSC of the integrated energy efficiency of the end user in smart industrial park through the index and layer. It is shown in Fig. 2. 


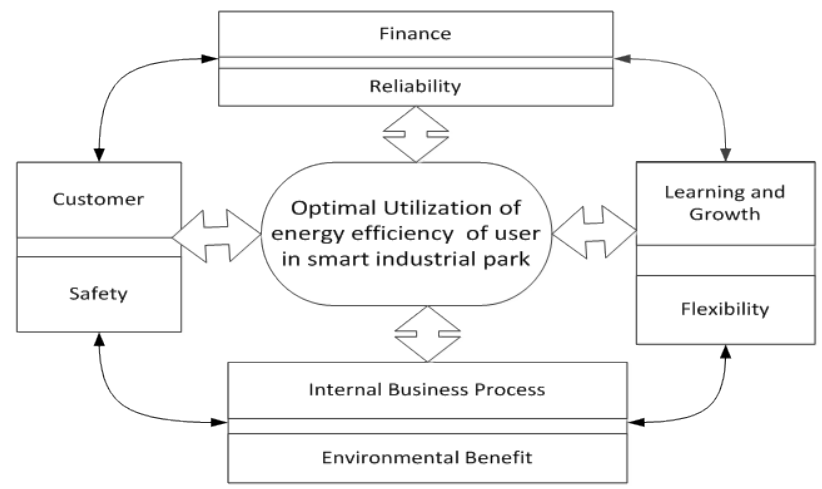

Fig. 2. BSC of integrated energy efficiency evaluation for smart industrial parks' user

When the index system is established, the weight of the index is the important part for the evaluation. The common calculation method of the weight has the subjective method, such as the two factor method, analytic hierarchy process, the expert investigation method and so on, and has the objective method, such as principal component analysis method, the multi objective programming method, the entropy and so on.

Compared with the traditional balanced score card method, in this paper it has mainly the following improvements: 1 ) in order to improve the expert scoring of judge summary, it changes 1-9 scale to 1 $\sim 3$ scale. 2) in order to improve the computational efficiency of the eigenvalue and eigenvector in the power method, it uses two standardizations for the convergence judgment, and accelerates the convergence speed. The calculation process of the improved BSC method is as follows.

\section{Step 1. The expert scoring}

The level distribution of the user's energy efficiency evaluation index is established. According to the distribution in Fig. 1, it is arranged in order form level 1 indicators, level 2 indicators and level 3 indicators. Then, the $\mathrm{N}$ experts are chosen to score on the same level of the index for the compair constract among any two index.

Step 2. The judgment Matrix A

Any expert scores the selected level indicators, in accordance with the Table 1 . The judgment matrix A of this index is established. It is shown in formulation (6).

$$
A=\left[\begin{array}{cccc}
a_{11} & a_{12} & \cdots & a_{1 n} \\
a_{21} & a_{22} & \cdots & a_{2 n} \\
\vdots & \vdots & \ddots & \vdots \\
a_{n 1} & a_{n 2} & \cdots & a_{n n}
\end{array}\right] \text { (6) }
$$

Where aij is the expert score of the compair constrast between the i-th index and the j-th index, $i=1,2 \ldots n, j=1,2 ; n, n$ is the number of indicators for the evaluation of the experts.

Table 1-Ratio Table of Index Important Degree

\begin{tabular}{llllll}
\hline Importance Degree & $\begin{array}{l}\text { the } \\
\text { same }\end{array}$ & more important & most important & less important & lest important \\
\hline Numerical value & 1 & 2 & 3 & $1 / 2$ & $1 / 3$ \\
\hline
\end{tabular}

Step 3. The eigenvalue and eigenvector

The improved power method is used for the maximum eigenvalue and eigenvector of the judgment matrix A. The calculation process is shown as follows.

Initialization 
Set a non-zero intilial vector V0. It generally is pre-selected $\mathrm{V} 0=\{1,1, \ldots, 1\}$, Then, the maximum value in $\mathrm{V0}$ is $\mathrm{f} 0$.

$$
f_{0}=\max \left\{\left|V_{0}\right|\right\}
$$

Iterative

According to the judgement matrix A, the iterative is done through the formulation (8).

$$
V_{k}=A * V_{k-1}
$$

Where $\mathrm{k}$ is from 1 to $\mathrm{N}$. The maximum value in the $\mathrm{Vk}$ is $\mathrm{m} 0$.

$$
\mathrm{m}_{0}=\max \left\{\left|\mathrm{V}_{k}\right|\right\}
$$

first standardization

The Vk is standardized in the first.

$$
V_{k}^{\prime}=V_{k} / \max \left\{\left|V_{k}\right|\right\}
$$

Where $\mathrm{k}$ is from 1 to $\mathrm{n}$. The maximum value in $V_{\mathrm{k}}^{\prime}$ is $\mathrm{m} 1 . \mathrm{m} 2$ is an average of $\mathrm{m} 0$ and $\mathrm{m} 1$.

$$
\begin{gathered}
m_{1}=\max \left\{\left|V_{k}^{\prime}\right|\right\} \\
m 2=(m 1+m 0) / 2
\end{gathered}
$$

second standardization

The $V_{\mathrm{k}}^{\prime}$ is standardized in the second.

$$
V_{k}^{\prime \prime}=V_{k}^{\prime} / \max \left\{\left|V_{k}^{\prime}\right|\right\}
$$

Where $\mathrm{k}$ is from 1 to $\mathrm{n}$. The maximum value in $V_{\mathrm{k}}^{\prime \prime}$ is $\mathrm{m} 3$. $\mathrm{f} 1$ is formulated in (15).

$$
\begin{gathered}
m_{3}=\max \left\{\left|\mathrm{V}_{k}^{\prime \prime}\right|\right\} \\
f_{1}=m_{0}-\frac{\left(m_{1}-m_{0}\right) *\left(m_{2}-m_{0}\right)}{m_{3}+m_{0}-m_{1}-m_{2}}
\end{gathered}
$$

convergence

The convergence accuracy $\varepsilon_{1}$ is $1 \times 10^{-6}$. If $f_{1}-f_{0}<\varepsilon_{1}, f_{1}$ is the approximate value of the eigenvalue of the judgment matrix A. $V_{k}$ is the eigenvector of the judgment matrix A. If not, $f_{0}=f_{1}$, and return b), continue。

Step 4. The consistency test.

If CR of the judgment matrix $A$ is less than 0.1 , it is considered that $A$ is feasible. If $C R$ is more than 0.1 , it is failure to the consistency test of A. Then, it will back to step 2, and constructs the qualified judgment matrix A again.

Step 5. The level weight of index

According to the selected $\mathrm{N}$ experts, respectively the step $2 \sim 4$ is done to obtain the eigenvector of each expert scoring. Then, the average of the eigenvector formulates in (16). The average weight determines the level 2 weights of the selected evaluation index.

$$
\overline{M_{i}}=\sum_{i=1}^{n} M_{i} / n
$$

Step 6. The total weight

In accordance with step $2 \sim 5$, the weight of the level 3 index is calculated. And the weight of index is set $\bar{Q}_{i}$, then the weight of the level 1 index is formulated in (17). 


$$
\bar{W}_{i}=\bar{M}_{i} * \bar{Q}_{i}
$$

Step 7. The scoring of the balanced scoring card

When the complete balanced score card is designed, Target score of each index has a set of weight index. Usually the set target is divided into 100 points. Then, the actual situation of each index were scored. The score of each index multiplies the corresponding weight of index, and the scores of all the indicators are summed. The sum is a value of the integrated energy efficiency evaluation. Finally, according to the scores from high to low order, it get the ranking of all the users in the region. The calculation processes such as shown in Fig. 3.

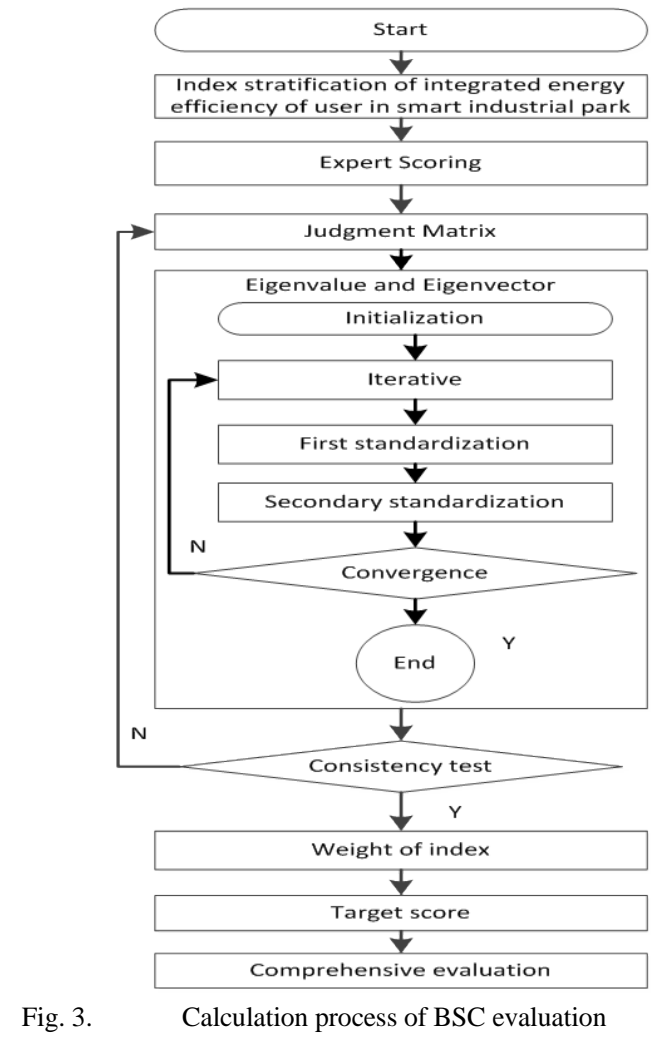

\section{Case Analysis}

\section{Case 1}

In order to verify the advantages of the improved balanced scorecard, the level 2 indicators of the integrated energy efficiency evaluation system of end user is applied. An expert is chosen to score for this indicators, which is shown in Table 2.

Table 2 - Expert scoring of level 2 indicators

\begin{tabular}{lllll}
\hline$M$ & $M_{1}$ & $M_{2}$ & $M_{3}$ & $M_{4}$ \\
\hline$M_{1}$ & 1 & 0.5 & 2 & 3 \\
$M_{2}$ & 2 & 1 & 3 & 3 \\
$M_{3}$ & 0.5 & 0.33 & 1 & 2 \\
$M_{4}$ & 0.33 & 0.33 & 0.5 & 1 \\
\hline
\end{tabular}

The data of the experts scoring is the input data, respectively, the improve the BSC method and the traditional BSC method is calculated. The weight of the level 2 indicators and the results are shown in Table 3. 
Table 3 - Weight and Results of Level 2 Indicators

\begin{tabular}{lll}
\hline$M$ & Improved BSC & BSC \\
\hline$W_{M 1}$ & 0.4570 & 0.4478 \\
$W_{M 2}$ & 0.1523 & 0.1547 \\
$W_{M 3}$ & 0.3006 & 0.2879 \\
$W_{M 4}$ & 0.0901 & 0.1096 \\
Iterative & 5 & 12 \\
Calculation Time(s) & 0.52 & 1.6 \\
\hline
\end{tabular}

It is obvious that the results is find out from Table 3

1) The order of the weight is WM3 $>$ WM2 $>$ WM $4>$ WM1. Comparing the improved BSC method and BSC method, the weight is not only little difference, but also is the completely consistent of the general trend.

2) The computational efficiency of the improved BSC method is faster than BSC. The iteration is greatly reduced, and the computational time is shortened.

Case 2

Table 4 -Expert Scoring of M1 Indicators

\begin{tabular}{llllllll}
\hline$M_{1}$ & & $M_{11}$ & $M_{12}$ & $M_{13}$ & $M_{14}$ & $M_{15}$ & $M_{16}$ \\
\hline \multirow{4}{*}{ Power Grid } & $M_{11}$ & 1 & 0.33 & 0.5 & 1 & 0.33 & 0.5 \\
& $M_{12}$ & 3 & 1 & 2 & 3 & 1 & 2 \\
& $M_{13}$ & 2 & 0.5 & 1 & 2 & 0.5 & 1 \\
& $M_{14}$ & 1 & 0.33 & 0.5 & 1 & 0.33 & 0.5 \\
& $M_{15}$ & 3 & 1 & 2 & 3 & 1 & 2 \\
& $M_{16}$ & 2 & 0.5 & 1 & 2 & 0.5 & 1 \\
\hline \multirow{4}{*}{ User } & $M_{11}$ & 1 & 2 & 0.5 & 1 & 3 & 0.5 \\
& $M_{12}$ & 0.5 & 1 & 0.33 & 0.5 & 1 & 0.33 \\
& $M_{13}$ & 2 & 3 & 1 & 2 & 3 & 1 \\
& $M_{14}$ & 1 & 2 & 0.5 & 1 & 2 & 0.33 \\
& $M_{15}$ & 0.33 & 1 & 0.33 & 0.5 & 1 & 0.33 \\
Government & $M_{16}$ & 2 & 3 & 1 & 3 & 3 & 1 \\
& $M_{11}$ & 1 & 3 & 2 & 1 & 3 & 3 \\
& $M_{12}$ & 0.33 & 1 & 0.5 & 0.33 & 1 & 0.5 \\
& $M_{13}$ & 0.5 & 2 & 1 & 0.5 & 3 & 1 \\
& $M_{14}$ & 1 & 3 & 2 & 1 & 3 & 3 \\
& $M_{15}$ & 0.33 & 1 & 0.33 & 0.33 & 1 & 0.5 \\
\hline
\end{tabular}

A typical case is a smart industrial park of Tianjin eco-city in China. It selects three aspect experts to score the integrated energy efficiency evaluation index of end users, which include the grid companies, users and government. The expert scorings is shown in Table $4 \sim 8$.

Table 5 - Expert Scoring of M2 Indicators

\begin{tabular}{lllll}
\hline$M_{2}$ & & $M_{21}$ & $M_{22}$ & $M_{23}$ \\
\hline \multirow{3}{*}{ Power Grid } & $M_{21}$ & 1 & 2 & 3 \\
& $M_{22}$ & 0.5 & 1 & 2 \\
& $M_{23}$ & 0.33 & 0.5 & 1 \\
\hline \multirow{3}{*}{ User } & $M_{21}$ & 1 & 0.5 & 2 \\
& $M_{22}$ & 2 & 1 & 3 \\
\multirow{3}{*}{ Government } & $M_{23}$ & 0.5 & 0.33 & 1 \\
& $M_{21}$ & 1 & 0.5 & 0.33 \\
& $M_{22}$ & 2 & 1 & 0.33 \\
\hline
\end{tabular}


Table 6 - Expert Scoring of M3 Indicators

\begin{tabular}{lllll}
\hline$M_{3}$ & & $M_{31}$ & $M_{32}$ & $M_{33}$ \\
\hline \multirow{3}{*}{ Power Grid } & $M_{31}$ & 1 & 0.5 & 2 \\
& $M_{32}$ & 2 & 1 & 3 \\
& $M_{33}$ & 0.5 & 0.33 & 1 \\
\hline \multirow{3}{*}{ User } & $M_{31}$ & 1 & 2 & 3 \\
& $M_{32}$ & 0.5 & 1 & 2 \\
& $M_{33}$ & 0.33 & 0.5 & 1 \\
\hline \multirow{3}{*}{ Government } & $M_{31}$ & 1 & 0.33 & 0.5 \\
& $M_{32}$ & 3 & 1 & 2 \\
\hline Table 7 - Expert Scoring of M4 Indicators & 2 & 0.5 & 1 \\
\hline$M_{4}$ & $M_{33}$ & $M_{41}$ & & $M_{43}$ \\
\hline \multirow{3}{*}{ Power Grid } & $M_{41}$ & 1 & 0.5 & 3 \\
& $M_{42}$ & 2 & 1 & 3 \\
\hline \multirow{3}{*}{ User } & $M_{43}$ & 0.33 & 0.33 & 1 \\
& $M_{41}$ & 1 & 3 & 0.5 \\
\hline \multirow{2}{*}{ Government } & $M_{42}$ & 0.33 & 1 & 1 \\
& $M_{43}$ & 0.5 & 2 & 0.5 \\
\hline
\end{tabular}

The data of the expert scorings is the input data, the improved BSC method is used for the integrated energy efficiency evaluation. The weight is shown in Table 8.

Table 8 - Weight Value of Assessment Index of Improved BSC Method

\begin{tabular}{lllll}
\hline Index & Weight of power grid & Weight of user & $\begin{array}{l}\text { Weight of } \\
\text { government }\end{array}$ & Total Weight \\
\hline$M_{11}$ & 0.0785 & 0.1147 & 0.1754 & 0.1229 \\
$M_{12}$ & 0.1342 & 0.0851 & 0.1113 & 0.1102 \\
$M_{13}$ & 0.1041 & 0.1527 & 0.0521 & 0.1030 \\
$M_{14}$ & 0.0478 & 0.0798 & 0.0298 & 0.0525 \\
$M_{15}$ & 0.0815 & 0.0541 & 0.0876 & 0.0744 \\
$M_{16}$ & 0.0621 & 0.0674 & 0.0510 & 0.0602 \\
$M_{21}$ & 0.0945 & 0.0652 & 0.0347 & 0.0648 \\
$M_{22}$ & 0.0801 & 0.0975 & 0.0757 & 0.0844 \\
$M_{23}$ & 0.0308 & 0.0548 & 0.0951 & 0.0602 \\
$M_{31}$ & 0.0951 & 0.0989 & 0.0741 & 0.0894 \\
$M_{32}$ & 0.1004 & 0.0478 & 0.1024 & 0.0835 \\
$M_{33}$ & 0.0601 & 0.0579 & 0.0804 & 0.0661 \\
$M_{41}$ & 0.0095 & 0.0147 & 0.0087 & 0.0110 \\
$M_{42}$ & 0.0201 & 0.0056 & 0.0045 & 0.0101 \\
$M_{43}$ & 0.0012 & 0.0038 & 0.0172 & 0.0074 \\
\hline
\end{tabular}

In Table 4 8, it is obvious as follow.

1) According to the weight of the experts, the different experts stress on different index. In M1, the power grid experts' emphasis on M12, the users' experts focus on M13, the government experts' emphasis on M11. In M2, the power grid experts' emphasis on M21, the users' experts focus on M22, the government experts' emphasis on M23. In M3, the power grid experts' emphasis on M31, the users' experts and the government experts' emphasis on M32. In M4, the power grid experts' emphasis on M42, the users' experts focus on M41, and the government experts' emphasis on M43. 
2) The top three of the weight can be known, the order of the power grid is M12>M13>M32, and the order of users is M13 $>$ M11 $>$ M31, the order of the government is M11 $>$ M12 $>$ M33. We infer that an energy supply enterprise should be the first choice for reliability and environmental benefits in the process of energy supply services.

\section{Conclusions}

In order to evaluate the integrated energy efficiency of end user in smart industrial park, an improved balanced score card method is proposed. The result of the evaluation can provide the basis for the energy supply enterprises, which carry out the energy management and operation analysis.

Next, combined with the existing energy efficiency evaluation and application, we can make full use of many new technologies, which include cloud computing, big data, Internet of things, Internet + , energy Internet and so on. It provides better platform and opportunity for the comprehensive utilization of user energy, and deserves further research.

\section{Acknowledgment}

The authors express sincere gratitude to the State Grid Science and Technology Project of China for providing financial support to carry out the research work. The name of the project is research on key technologies of energy framework of smart grid in smart city.

\section{References}

1. J.P. Zhang, J.J. Hu, Design of Smart Grid Oriented Adaptive Planning System, Automation of Electric Power Systems, 2011, vol. 35(10), pp. 1-7.

2. X.B. Yang, H.M. Li, Z.D. Yin, et al. Energy Efficiency Index System for Distribution Network Based on Analytic Hierarchy Process, Automation of Electric Power System, vol. 37(21), pp. 146150,195, 2013.

3. L.J. Ge, S.X.Wang, M.Zhang, et al. Power usage management \& service platform in smart electricity utilization condition, Electric Power Automation Equipment, vol. 35(3), pp. 152-156. 2015.

4. Y.M. Luo, L.F. Mao, J.G. Yao, et al. Evaluation Model of Integrated Energy Efficiency for Power Users, Proceedings of the CSU-EPSA, vol. 23(5), pp. 104-109. 2011.

5. Z.Y. Qu, C. Wang, L. Wang, et al. Fuzzy comprehensive evaluation of household electric energy consumption, Advanced Technology of Electrical Engineering and Energy, vol. 34(6), pp. 57-63. 2015.

6. H.P. Tian, S.H. Du, X.H. Han, et al. Assessment of Power Integrated Energy Efficiency for Industrial Enterprise Users Based on AHP-entropy Method, Smart Grid, vol. 3(2), pp. 112-118. 2015. 7. R.J. Zhou, X.H. Ran, F.L. Mao, et al. Energy-Saving Coordinated Optimal Dispatch of Distributed Combined Cool, Heat and Power Supply, Power System Technology, vol. 36(6), pp. 814. 2012.

8. Y.J. Fang, M. Tang, and W. Huang, Electricity energy efficiency rating assessment method for high -consuming enterprises, Electrical Measurement \& Instrumentation, vol. 52(6), pp. 6-10,54. 2015.

9. Y.B. Shu, and J.B. Fan, Progress in Smart City Standardization, Power System Technology, vol. 38(10), pp. 2617-2623. 2014. 
10. A.Mojtaba, N. Esmaeil, T.M. Reza, et al. A network based data envelope analysis model in a dynamic balanced score card, Mathematical Problems in Engineering, vol. 23, pp. 1-14. 2015.

11. S. Maria, L.G. Maite, F. Emilio, et al. Ten years of pre-analytical monitoring and control: synthetic balanced score card indicator, Biochemia Medica, vol. 25(1), pp. 49-56. 2015.

12. J. Neda, A.B. Nooh, and T. Hamed, Propose a methodology to implement balanced score card for operational appraisal of industrial groups, Procedia Technology, vol. 12, pp. 659-666. 2014.

13. K. Vladimir, F. Galina, S.F. Aleksandrs, et al. Innovative Nuclear Energy Systems: State of the Art Survey on Evaluation and Aggregation Judgment Measures Applied to Performance Comparison, Energies, vol. 8, pp. 3679-3678, 2015.

14. E.G. Zhu, L.J. Dong, L.J. Ge, et al. Interval entropy method for user's electric equipment evaluation, Electrical Measurement \& Instrumentation, vol. 51(24) , pp. 106-112, 2014.

15. M. Yao, D.L. Jiang, and G.Y. Chen, Application of Fuzzy Clustering on Power Grid Harmonic Comprehensive Evaluation, Electrical Measurement \& Instrumentation, vol. 48(550), pp. 1-4, 24, 2011 